\title{
Application of novel targeted molecular imaging probes in the early diagnosis of upper urinary tract epithelial carcinoma
}

\author{
GUODONG LIAO $^{1}$, LIJIANG WANG ${ }^{2}$ and WEIWEN YU ${ }^{1}$ \\ ${ }^{1}$ Department of Urology, Zhejiang Province People's Hospital; \\ ${ }^{2}$ Zhejiang-California International NanoSystems Institute, Hangzhou, Zhejiang 310014, P.R. China
}

Received February 6, 2017; Accepted August 10, 2018

DOI: $10.3892 / \mathrm{ol} .2018 .9430$

\begin{abstract}
Imaging techniques of upper tract urothelial carcinoma (UTUC) are presently limited. Upconversion particles (UCPs) could be used to target tumors for imaging. The present study aimed to assess the value of a nano-UCP as a diagnostic probe for deep tumor tissue, including UTUC. Polymer-coated water-soluble UCPs were synthesized. The $\mathrm{pH}$ Low Insertion Peptide (pHLIP) polypeptide was synthesized using the solid phase method. The silane shell surface was modified to present amino or carboxyl groups. Succinimidyl 4-(N-maleimidomethyl)cyclohexane-1-carboxylate was used for the coupling of the polypeptide to the UCPs. An animal model of subcutaneous tumor was established in 4-week old nude mice using UTUC cells. Urinary tract epithelial cancer $\mathrm{T}_{24}$ cells were injected into the diaphragm below the heart. PHLIP-UCP solution ( $1 \mathrm{ml}$ ) was injected into the abdominal cavity of each animal. Optical detection was performed using a small animal living body multispectral imaging system. UCPs dispersed in chloroform emitted no light under natural light, while they emitted a green light when excited with a 980-nm laser. The maximum emission wavelength of $\mathrm{Ho}^{3+}$-doped UCPs was $\sim 550 \mathrm{~nm}$ and the red emission region was $\sim 650 \mathrm{~nm}$. As the coated UCPs possessed a tendency to agglomerate and precipitate, the yield of the UCPs in the aqueous phase was reduced. Tumors could be successfully imaged in tumor-bearing mice. $\mathrm{NaYF}_{4}$ : $\mathrm{Yb}, \mathrm{Ho}^{3+} \mathrm{UPC}$ could be used for the detection of UTUC, thus further studies are
\end{abstract}

Correspondence to: Dr Weiwen Yu, Department of Urology, Zhejiang Province People's Hospital, 1 Shangtang Road, Hangzhou, Zhejiang 310014, P.R. China

E-mail:hfhyww@sina.com

Abbreviations: CTU, computed tomography urography; EDC, 1-ethyl-3-(3-aminopropyl-methyl-2) carbon imine; MRI, magnetic resonance imaging; NHS, N-hydroxysuccinimide; NIRF, near-infrared fluorescence; TEM, transmission electron microscopy; UCP, upconversion particles; UTUC, upper tract urothelial carcinoma

Key words: upper tract urothelial carcinoma, upconversion particles, near-infrared fluorescence imaging required to determine if it could be used in larger animals with deeper tumors.

\section{Introduction}

Upper tract urothelial carcinoma (UTUC) originates from the ureter and renal pelvis. UTUC are rare and account for only $5-10 \%$ of urothelial cancers $(1,2)$. The incidence of UTUC in western countries is about 2 per 100,000 person-year $(1,2)$. The most common symptoms are hematuria in $70-95 \%$ of cases (3). Due to the deep location of the ureter and kidney, and because the muscle layer is not as thick as in the bladder, the diagnosis of upper urinary tract epithelial cancer is often made at a later stage than bladder cancer, resulting in poor overall prognosis (4). In addition, $\sim 60 \%$ of upper urinary tract epithelial cancers are invasive at diagnosis, compared with $15 \%$ for bladder cancer $(5,6)$. Therefore, the early diagnosis of upper urinary tract epithelial cancer is an important factor affecting the survival rate of patients (4).

At present, the diagnosis of UTUC mainly involved computed tomography urography (CTU), magnetic resonance imaging (MRI), cystoscopy and urinary cytology, and diagnostic ureteroscopy (4). Urine cytology is highly specific and non-invasive, and some authors believe that it is the gold standard for follow-up of tumor recurrence (7). However, the sensitivity of this method is poor and specimen collection, inflammation, stones, and foreign bodies, can affect the results of the examination (4). CTU is preferable to MRI because of better accuracy and lower costs (4), but CTU is unable to detect flat lesions unless they create a mass effect or urothelial thickening (8). Finally, ureteroscopy is relatively invasive, but may be useful when diagnosis is uncertain, when conservative treatment is considered, or when the patient has a single kidney (4). Therefore, traditional diagnosis methods have a number of limitations.

Novel methods include photodynamic diagnosis of blood porphyrin derivatives that accumulate in tumor tissue (9). However, these techniques show not only UTUC, but also hyperplasia of the transitional epithelium, squamous metaplasia, inflammation, and granulation tissue $(10,11)$. In addition, other factors may also affect the efficacy of these methods including bladder retention time, different display equipment, facilities, light intensity, cystoscopy observation angle, physician experience (at least 15 fluorescence cystoscopy experience) (10), and preoperative drugs or bladder perfusion therapy (12). 
In recent years, near-infrared fluorescence (NIRF) imaging received more attention from researchers. Light at $600-1,100 \mathrm{~nm}$ has a strong tissue penetration, but there is a strong background of spontaneous fluorescence $(13,14)$. Upconversion luminescence is a phenomenon that emits visible light under the excitation of infrared light $(13,14)$. So far, most of the research on upconversion particles (UCP) was mainly performed using rare earth metal particles. Novel molecular imaging probes with high sensitivity, high stability of fluorescence, good safety, and high energy conversion of light (known as anti-Stokes effect) are now suitable for NIRF imaging (13,14). Chatterjee et al (15) published the first report about NaYF4: YbUCP coated with Er that were used for imaging of tumor cells and small animals. Their results showed that these UCPs had good biocompatibility and no toxicity to bone marrow stem cells (15). However, to be able to detect cancer cells specifically, surface functionalization of the nanoparticles is needed, which is usually achieved by coupling antibodies. Although numerous studies have attempted to create UCPs with rare earth ions, the feasibility of tumor cells in vivo imaging still need further study (16-18).

Therefore, the aim of the present study was to assess the value of a nono-UCP as a diagnostic probe for deep tumor tissue like UTUC. These results could pave the way for new technologies for the detection of UTUC.

\section{Materials and methods}

Synthesis of polymer-coated water-soluble UCP. All rare earth reagents used in this study were $5 \mathrm{~N}(99.999 \%)$. In a beaker, a solution containing $1.2 \mathrm{mmol} \mathrm{NaCl}, 0.48 \mathrm{mmol} \mathrm{YCl}_{3}$, $0.108 \mathrm{mmol} \mathrm{YbCl}_{3}, 0.012 \mathrm{mmol} \mathrm{HoCl}$ (Aladdin Chemicals Co., Ltd., Shanghai, China), $0.15 \mathrm{~g}$ of PEI was prepared in $15 \mathrm{ml}$ of ethylene glycol. After stirring on a magnetic stirrer, $0.4 \mathrm{~g}$ of $\mathrm{NaF}$ was added, the solution was stirred for $10 \mathrm{~min}$. The reaction kettle was sealed into a muffle furnace and heated for $2 \mathrm{~h}$ at $200^{\circ} \mathrm{C}$. After slow cooling to room temperature, the precipitate was collected by centrifugation. The pellet was washed three times with water and ethanol. After drying, the nanoparticles were dispersed in water. The particle size of the UCPs was determined by dynamic light scattering and transmission electron microscopy (TEM) on a JEOL/JEM-2010F TEM (JEOL, Tokyo, Japan). Infrared spectroscopy was used to analyze the surface of the UCPs. The luminescence spectra of the particles were determined by fluorescence spectrometer using an external 980-nm light source.

Coupling of $p H$ Low Insertion Peptide ( $p H L I P$ ) with UCP. The pHLIP polypeptide was synthesized by the solid phase method and using the following sequence: AlaCysGluGlnAsn ProIleTyrTrpAlaArgTyrAlaAspTrpLeuPheThrThrProLeuLeu LeuLeuAspLeuAlaLeuLeuValAspAlaAspGluGlyThrGly.

Aqueous phase transfer is the first prerequisite for biomedical applications of UCPs, but ifyou need to use UCPs to achieve specific functions, for example, targeted imaging or light detection for specific objects, it is necessary to carry out surface modification of UCP, which is usually achieved through coupling antibodies or other targeting molecules. The silane treatment makes the particles hydrophilic, its surface mainly presenting hydroxyl groups, which cannot directly link proteins. Therefore, the silane shell surface was modified to present amino or carboxyl groups by the crosslinking agent 1-ethyl-3-(3-aminopropyl-methyl-2) carbon imine (EDC) and two N- hydroxysuccinimide (NHS) reactions.

The linker (SMCC; Pierce; Thermo Fisher Scientific, Inc., Waltham, MA, USA) was used for the coupling of the polypeptide to the particles through the Cysthiol group and the particle surface amino group at the $\mathrm{N}$ terminal side of the peptide. Cy5-labeled pHLIP was used as positive control, and unlabeled UCP was used as negative control. Anurinary tract epithelial cancer cell line cultured in vitro was used as a model. The cell binding reaction of pHLIP-UCP was observed at $\mathrm{pH}$ 5-7. The experiments were repeated to determine the optimal pHLIP-UCP coupling conditions.

For linking proteins to nanoparticles using SMCC, $50 \mathrm{mg}$ of SMCC was dissolved in $5 \mathrm{ml}$ of DMF to obtain a solution at $10 \mathrm{mg} / \mathrm{ml}$. High-brightness purified UCPs were added to obtain a solution at about $0.015 \mathrm{mmol}$ and $5 \mathrm{ml}$ of phosphate-buffered saline were added and mixed quickly. The solution was left to react for $30 \mathrm{~min}$ at $37^{\circ} \mathrm{C}$. Excess SMCC was removed by PBS dialysis, $1 \mathrm{ml}$ of $0.15 \mathrm{M}$ of pHLIP polypeptide was added, and the reaction was continued at $37^{\circ} \mathrm{C}$ for $30 \mathrm{~min}$. After a period of silane reaction, the clear solution gradually became milky white, indicating that the silane carried on the coupling reaction on the surface of the particles. The coated particles gradually precipitated the organic phase. In order to observe the continuous process of silane modification to determine the optimal modification time, samples were observed after $12,24,36,42$, and $48 \mathrm{~h}$ by TEM. The nanoparticles were obtained after centrifugal precipitation and repeated washing. Nanoparticles were kept at $4^{\circ} \mathrm{C}$.

High-sensitivity imaging of early stage urinary tract cancer tumor model using pHLIP-UCP. An animal model of subcutaneous tumor was established in 4-week old nude mice using UTUC cells. All applicable institutional and national guidelines for the care and use of animals were followed. The tumors were observed from the first day after inoculation. pHLIP-Cy5.5 was used as the positive control, prepared as previously described (19). Uncoupled UCPs were used as the negative control. A foreign body was implanted at the side of the inoculation site to cause local inflammation and to observe the effect of inflammation on the sensitivity of the probe.

Cultured urinary tract epithelial cancer cell line $\mathrm{T}_{24}$ was cultured. About $0.5 \times 10^{6}$ cells in $0.1 \mathrm{ml}$ were injected in the diaphragm below the heart. PHLIP-UCP solution $(1 \mathrm{ml})$ was injected in the abdominal cavity of each animal.

Optical detection was performed using a small animal living body multi spectral imaging system (Maestro; CRi, Inc., Woburn, MA, USA). The spectral detection range was 400-950 $\mathrm{nm}$. The excitation source (infrared laser diode) was set at $980 \mathrm{~nm}$ and the power was $0.2 \mathrm{~W}$ (Changchun Mingwan Optics Co., Changchun, China) (20). The image was captured by a CCD camera (Andor Clara with Sony ICX285 CCD; Sony, Connecticut, NE, USA). Images were analyzed using the Maestro software analysis system 2.4 (CRi, Inc.). Fluorescence intensity was determined to represent the enrichment degree and sensitivity of the probe to the tumor cells. 


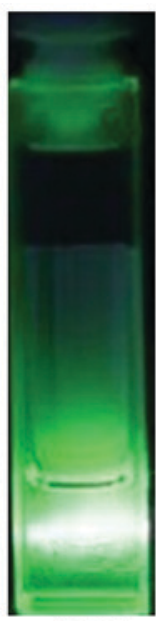

Nano

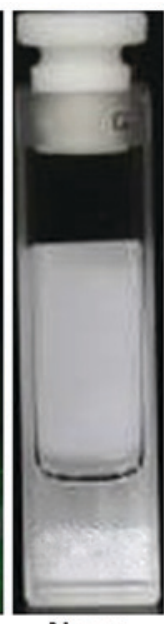

Nano

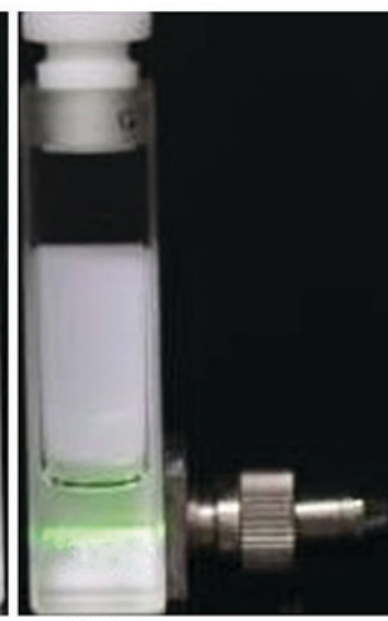

Nano
Figure 1. UCP chloroform solution under the excitation of natural light and 980-nm laser. UCPs dispersed in chloroform emit no light under natural light (center vial), while they emit a green light when excited with a 980-nm laser (left vial). UCP, upconversion particle.
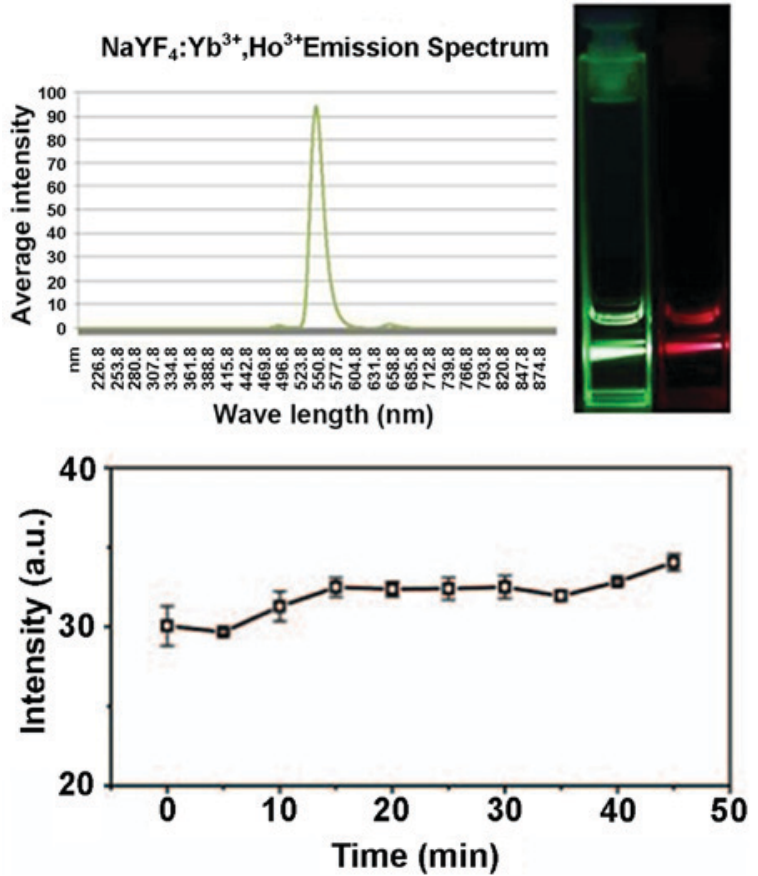

Figure 2. $\mathrm{Ho}^{3+}$-doped UCP spectra, laser induced graphs, and upconversion luminescence duration. Recorded at a wavelength of $980 \mathrm{~nm}$ after $2 \mathrm{~min}$ of excitation, each data point corresponds to an average duration of luminous intensity. UCP, upconversion particle.

\section{Results}

Synthesis of UCP. Fig. 1 shows that the UCPs dispersed in chloroform emitted no light under natural light (center vial), while they emitted a green light when excited with a 980-nm laser (left vial).

Excitation of $\mathrm{Ho}^{3+}$-doped UCP. The spectra presented in Fig. 2 were captured using green and red filters. The graph shows that the emission of the excitation channel was uniform and transparent and the intensity was high. The maximum emission wavelength of $\mathrm{Ho}^{3+}$-doped UCPs was about $550 \mathrm{~nm}$ and the red emission region was around $650 \mathrm{~nm}$. When the doping was $2 \%$, the green luminescence dominates and the proportion of red emission was increased when the proportion of active ions was increased.

Phase transfer of $\mathrm{NaYF}_{4}: \mathrm{Yb}, \mathrm{Ho}^{3+} \mathrm{UPCs}$. The diameter of the UCPs was $10.9 \pm 1.7 \mathrm{~nm}$. They had a round shape. The $\mathrm{NaYbF}_{4}$ particles had a crystal structure of about $0.38 \mathrm{~nm}$ composed of a planar hexagon. The surface of $\mathrm{NaYF}_{4}$ : $\mathrm{Yb}, \mathrm{Ho}^{3+} \mathrm{UPCs}$ was coated with a uniform layer of silica. Fig. 3 shows that the UCPs dispersed evenly in aqueous solution and that the surface of the particles was coated with peptides to achieve the function of the UPCs. A continuous process of silane reaction is shown in Fig. 3. It can be seen that the UCPs were uniformly coated with a layer of silane and that the shell became gradually thicker with elapsing time.

Functional modification of the NaYF4: $\mathrm{Yb}, \mathrm{Ho}^{3+}$ UPCs. As shown in Fig. 4, the solution is transparent in aqueous solution. Because the coated UCPs had a tendency to agglomerate and precipitate, the yield of the UCPs in the aqueous phase was reduced and the overall fluorescence intensity of the aqueous phase particles was reduced. Compared with the corresponding organic phase solution, the fluorescence intensity was decreased.

Imaging of tumor bearing animal model by UCPs. The nanoparticles were injected into the frontal chest and diaphragm (below the heart) of the mice. Fig. 5A shows the mice and tumor imaging after UCP injection. Fig. 5B shows that the tumor is visible with a visible $550 \mathrm{~nm}$ upconversion fluorescence emission. By analyzing the spectral slices at $550 \mathrm{~nm}$, as shown in Fig. 5C, the fluorescence intensity measurement is carried out by selecting the fluorescence emission region and the surrounding background region. The average counts signal is obtained and the average intensity signal is plotted over the corresponding region. Regions 3 and 4 represent the upconversion fluorescence region while regions 1 and 2 represent the background region. Taken from the ventral side, the image shows a signal-to-noise ratio of 18.5. Fig. 5D shows that the angle of view is important. Indeed, when imaged from the side, the ribs interfere with the laser and the emitted light, resulting in weaker signal. This can be overcome by increasing exposure time, but the background noise is increased. Spectral analysis of this region, as shown in Fig. 5E, shows that the $550-\mathrm{nm}$ fluorescence emission is very close to the background. By analyzing the fluorescence intensity of the $550 \mathrm{~nm}$ spectra, as shown in Fig. 5F, the signal-to-noise ratio is 2.08 , showing that the signal is still positive. Fig. $5 \mathrm{G}$ shows the relative location map of the conversion fluorescence emission for deep injection, which is expressed in the form of intensity.

\section{Discussion}

The imaging of UTUC is presently limited. UCPs could be used to target tumors for imaging. Therefore, this study aimed to assess the value of a nano-UCP as a diagnostic probe for deep tumor tissue like UTUC. Results showed that UCPs dispersed in chloroform emitted no light under natural light, while they 
A

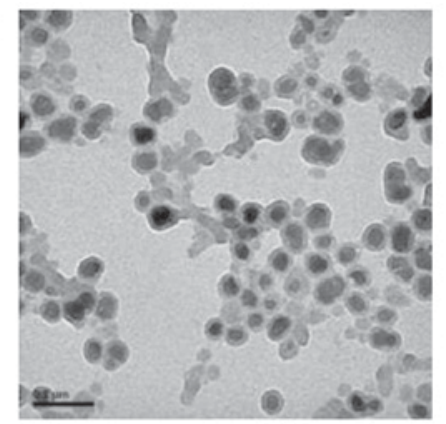

C

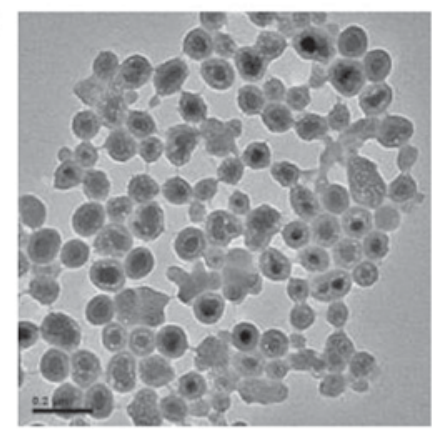

E

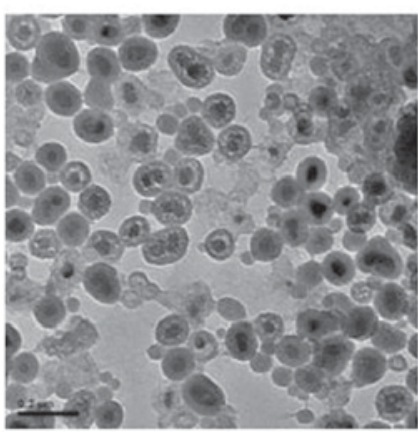

B

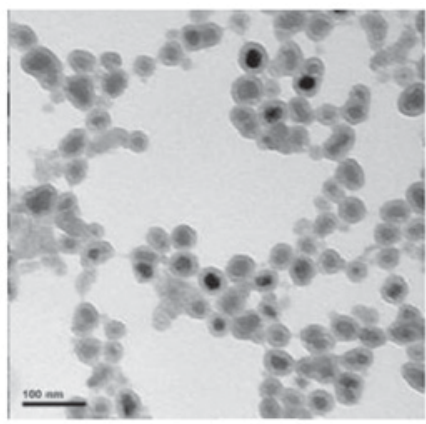

D

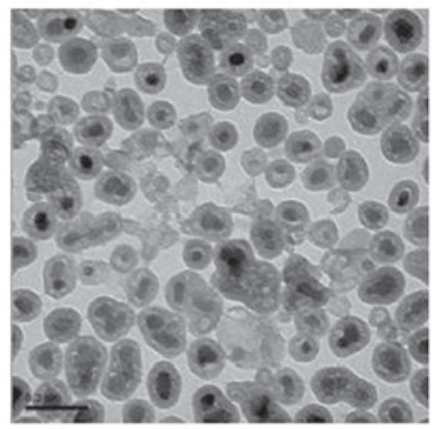

$\mathbf{F}$

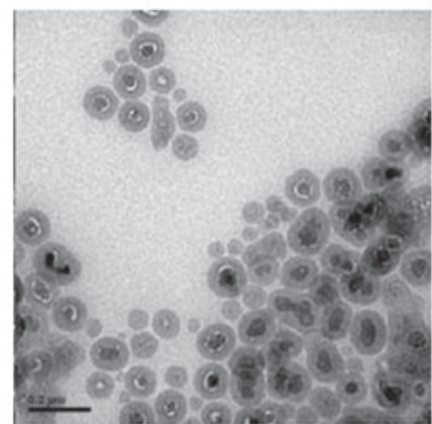

D $\quad E \quad F$

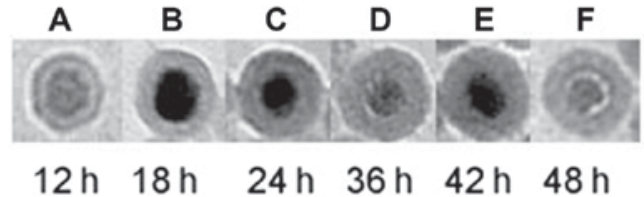

Figure 3. Continuous process of UCPs modified by silane. It can be seen that the UCPs were uniformly coated with a layer of silane and that the shell became gradually thicker with elapsing time. (A) 12 h; (B) 18 h; (C) 24 h; (D) 36 h; (E) 42 h; (F) 48 h. UCP, upconversion particle.

emitted a green light when excited with a 980 -nm laser. The maximum emission wavelength of $\mathrm{Ho}^{3+}$-doped UCPs was about $550 \mathrm{~nm}$ and the red emission region was around $650 \mathrm{~nm}$. Because the coated UCPs had a tendency to agglomerate and precipitate, the yield of the UCPs in the aqueous phase was reduced. Tumors could be successfully imaged in tumor-bearing mice. These results suggest that $\mathrm{NaYF}_{4}: \mathrm{Yb}, \mathrm{Ho}^{3+} \mathrm{UPCs}$ could be used for the detection of UTUC.

For in vivo imaging, the probe has to be resistant to light, possess a good dispersion, and be biocompatible. In order to achieve these features, the present study used a process that first involves the preparation of the nanoparticles, which are then doped with $\mathrm{Yb}^{3+} / \mathrm{Ho}^{3+}$ doped with $\mathrm{NaYF}_{4}$, as previously described (21). Thereafter, the surface is coated with a non-porous silica shell. Finally, the UCPs were coupled with peptides specific to UTUC cells. The final characteristics of the UCPs was similar to previously published results (22).

At room temperature, using an excitation wavelength of $980 \mathrm{~nm}$, the strongest emission wavelength was at $\sim 550 \mathrm{~nm}$,

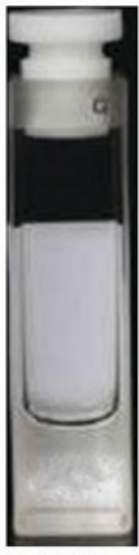

UCP

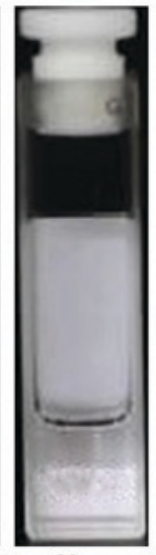

Nano

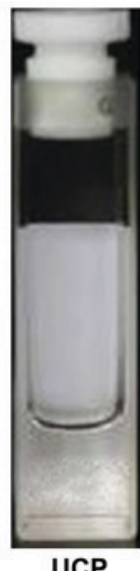

UCP
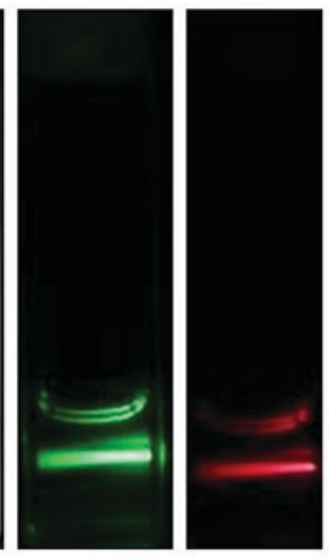

Figure 4. Degree of dispersion and fluorescence of the functionally modified UCPs in the aqueous phase. Because the coated UCPs had a tendency to agglomerate and precipitate, the yield of the UCPs in the aqueous phase was reduced and the overall fluorescence intensity of the aqueous phase particles was reduced. Compared with the corresponding organic phase solution, the fluorescence intensity was decreased. UCP, upconversion particle. 
A a

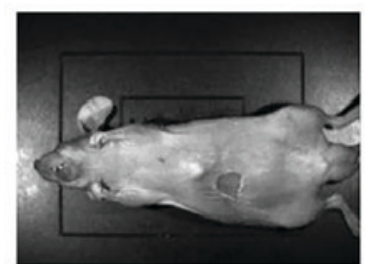

B a

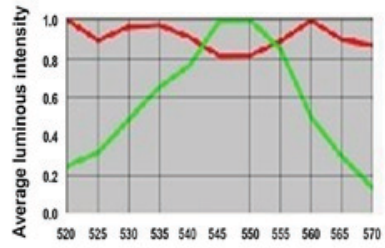

Wave length $(\mathrm{nm})$

C a

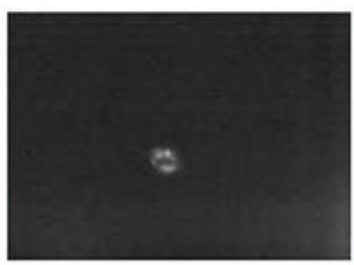

D a

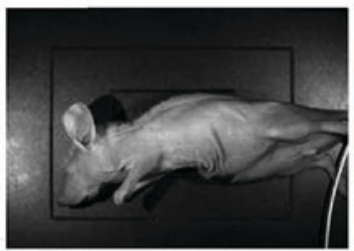

E a

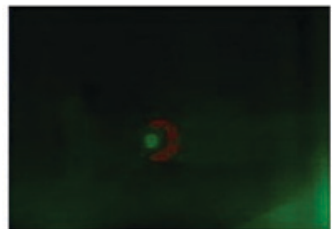

F a

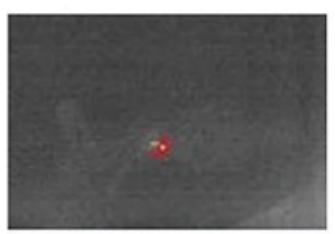

G a

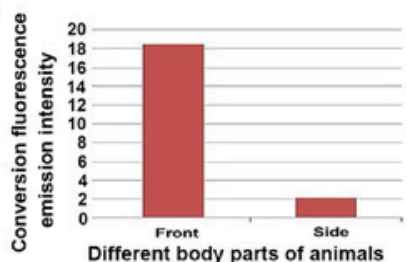

b

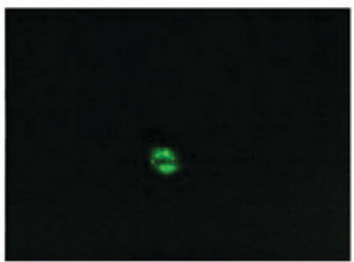

b

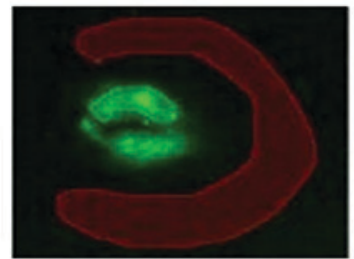

b
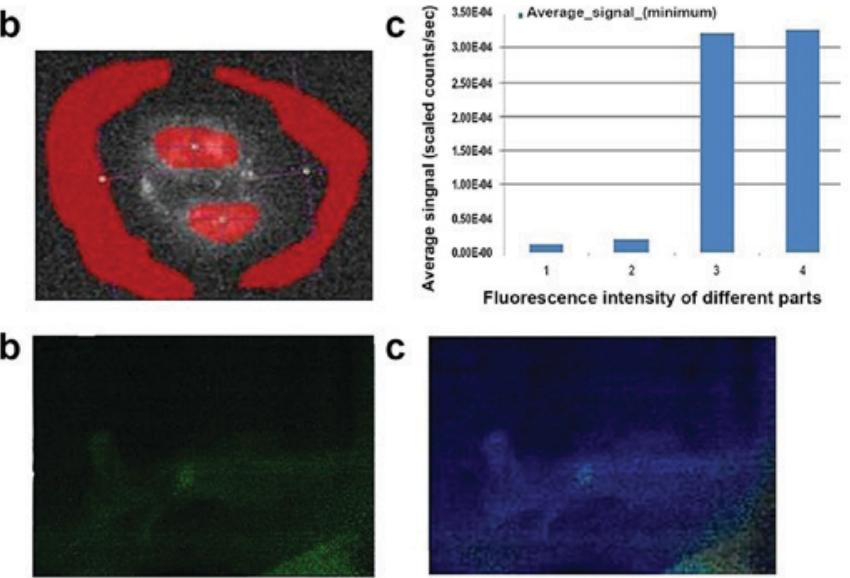

b

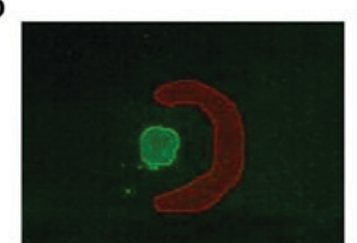

b

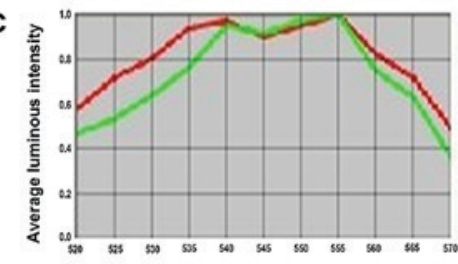

c

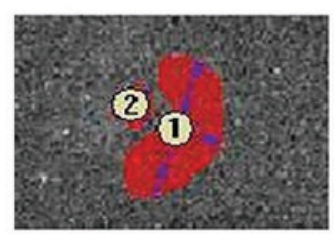

b

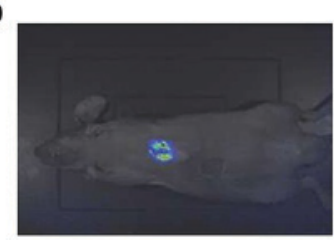

g

Wavo length (nm)

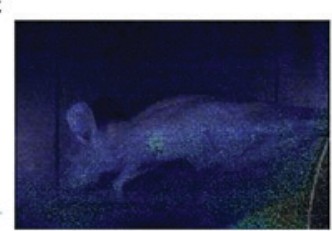

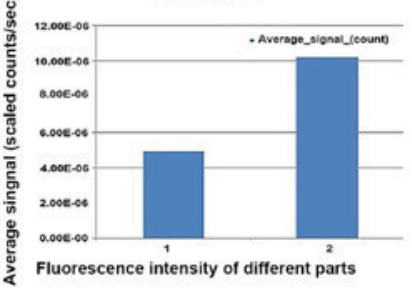

Figure 5. Fluorescence spectrum analysis of imaging fluorescence in transplanted tumors. (A) Imaging of the mice and tumor from the ventral angle. (B) Analysis of the tumor imaged using UCPs. The green area is the tumor and the red area is the background. (C) Analysis of the fluorescence intensity at $550 \mathrm{~nm}$. The signal-to-noise ratio was 18.5. (D) Imaging of the mice and tumor from the side angle. (E) Analysis of the tumor imaged using UCPs from the side angle. The green area is the tumor and the red area is the background. $(\mathrm{F})$ Analysis of the fluorescence intensity at $550 \mathrm{~nm}$. The signal-to-noise ratio was 2.08. (G) Relative location map of the conversion fluorescence emission for deep injection, which is expressed in the form of intensity. UCP, upconversion particle.

which may be due to the generation of the $\mathrm{Ho}^{3+}$ to the $5 \mathrm{I} 8$ transition of 5S2 (23). At the same time, results showed that the particles emitted a strong signal for as long as $40 \mathrm{~min}$. These properties make these particles suitable for use in a clinical setting.
Nanoparticles may enter the cells via passive absorption and active uptake pathway. Unfortunately, these entry patterns have great limitations in the diagnosis of cancers because of the lack of specificity of the nanoparticles to cancer cells. Therefore, the nanoparticles have to becoupled with molecules that targetcancer 
cells. In the present study, the UCPs were coupled to pHLIP, which is a peptide that can recognize acidic cancer cells (24-26). Therefore, after coupling the molecular probe with the nanoparticles, the biological activity of the probe is changed. A tumor host interface microenvironment is formed after the interaction between tumor cells and adjacent host components (27). The growth rate of tumor cells is fast and the metabolism rate is high, which leads to an acidic microenvironment, which is an important feature of tumor biology (28).

The results of the present study showed that pHLIP-bound UCPs could be used successfully to image tumors in mice. However, mice are small animals that are easy to image using NIRF imaging. Future studies will have to test this modality in larger animals, in which the tumors will be deeper. Indeed, the main obstacle to optical imaging for biological and medical applications isthat most biological tissues can absorb or scatter light. NIRF imaging, which includes upconversion luminescence, needs to pay more attention to better tissue penetration and low background fluorescence. This problem directly affects the quality of the detected signal and the accuracy of the image. Further study is necessary to improve this technology before its clinical applications.

In this study, upconversion luminescence was used to establish nanoprobes for imaging of living animals, without background fluorescence. The probe had a high signal-to-noise ratio and could achieve tissue imaging. $\mathrm{NaYF}_{4}: \mathrm{Yb} \mathrm{Ho}^{3+} \mathrm{UPCs}$ could be used for the detection of UTUC. Further studies are necessary to determine if it could be used in larger animals with deeper tumors.

\section{Acknowledgements}

The present study was supported by the Public Welfare Technology Application Research Projects of Zhejiang Provincial Science and Technology Agency (no. 2014C33129).

\section{Competing interests}

The authors declare that they have no conflict of interest.

\section{References}

1. Munoz JJ and Ellison LM: Upper tract urothelial neoplasms: Incidence and survival during the last 2 decades. J Urol 164: 1523-1525, 2000.

2. Siegel R, Naishadham D and Jemal A: Cancer statistics, 2012. CA Cancer J Clin 62: 10-29, 2012.

3. Cowan NC: CT urography for hematuria. Nat Rev Urol 9: 218-226, 2012

4. Rouprêt M, Zigeuner R, Palou J, Boehle A, Kaasinen E, Sylvester R, Babjuk M and Oosterlinck W: European guidelines for the diagnosis and management of upper urinary tract urothelial cell carcinomas: 2011 update. Eur Urol 59: 584-594, 2011.

5. Babjuk M, Oosterlinck W, Sylvester R, Kaasinen E, Böhle A, Palou-Redorta $\mathrm{J}$ and Rouprêt M; European Association of Urology (EAU): EAU guidelines on non-muscle-invasive urothelial carcinoma of the bladder, the 2011 update. Eur Urol 59: 997-1008, 2011.

6. Margulis V, Shariat SF, Matin SF, Kamat AM, Zigeuner R, Kikuchi E, Lotan Y, Weizer A, Raman JD and Wood CG; The Upper Tract Urothelial Carcinoma Collaboration: Outcomes of radical nephroureterectomy: A series from the Upper Tract Urothelial Carcinoma Collaboration. Cancer 115: 1224-1233, 2009.

7. Oosterlinck W: Guidelines on diagnosis and treatment of superficial bladder cancer. Minerva Urol Nefrol 56: 65-72, 2004.
8. Xu AD, Ng CS, Kamat A, Grossman HB, Dinney C and Sandler CM: Significance of upper urinary tract urothelial thickening and filling defect seen on MDCT urography in patients with a history of urothelial neoplasms. AJR Am J Roentgenol 195: 959-965, 2010.

9. Stepp H, Baumgartner R, Kriegmair $M$ and Hofstetter A: Fluorescence diagnosis of bladder tumor using 5-Aminolevulinic acid-fundamentals and results. Verlag Endo-Press, Berlin, 2000.

10. De Dominicis C, Liberti M, Perugia G, De Nunzio C, Sciobica F, Zuccalà A, Sarkozy A and Iori F: Role of 5-aminolevulinic acid in the diagnosis and treatment of superficial bladder cancer: Improvement in diagnostic sensitivity. Urology 57: 1059-1062, 2001.

11. Zaak D, Kriegmair M, Stepp H, Stepp H, Baumgartner R, Oberneder R, Schneede P, Corvin S, Frimberger D, Knüchel R and Hofstetter A: Endoscopic detection of transitional cell carcinoma with 5-aminolevulinic acid: Results of 1012 fluorescence endoscopies. Urology 57: 690-694, 2001.

12. Grimbergen MC, van Swol CF, Jonges TG, Boon TA and van Moorselaar RJ: Reduced specificity of 5-ALA induced fluorescence in photodynamic diagnosis of transitional cell carcinoma after previous intravesical therapy. Eur Urol 44: 51-56, 2003.

13. Soukka T, Kuningas K, Rantanen T, Haaslahti V and Lövgren T: Photochemical characterization of up-converting inorganic lanthanide phosphors as potential labels. J Fluoresc 15: 513-528, 2005.

14. Vennerberg D and Lin Z: Upconversion nanocrystals: Synthesis, properties, assembly and applications. Sci Adv Mater 3: pp26-40, 2011.

15. Chatterjee DK, Rufaihah AJ and Zhang Y: Upconversion fluorescence imaging of cells and small animals using lanthanide doped nanocrystals. Biomaterials 29: 937-943, 2008.

16. Cheng L, Yang K, Zhang S, Shao M, Lee S and Liu Z: Highly sensitive multiplexed in vivo imaging using pegylated upconversion nanoparticles. Nano Res 3: pp722-732, 2010.

17. Cheng L, Yang K, Shao M, Lee ST and Liu Z: Multicolor in vivo imaging of upconversion nanopaticles with emissions tuned by luminescence resonance energy transfer. J Phys Chem 115: pp2686-2692, 2011.

18. Liu Q, Chen M, Sun Y, Chen G, Yang T, Gao Y, Zhang X and Li F: Multifunctional rare-earth self-assembled nanosystem for tri-modal upconversion luminescence/fluorescence/positron emission tomography imaging. Biomaterials 32: 8243-8253, 2011.

19. Hahn CD, Riener CK and Gruber HJ: Labeling of antibodies with Cy3-, Cy3.5-, Cy5- and Cy5.5-monofunctional dyes at defined dye/protein ratios. Single Mol 2: 149, 2001.

20. Yi GS and Chow GM: Water-soluble NaYF4: $\mathrm{Yb}, \mathrm{Er}(\mathrm{Tm}) / \mathrm{NaYF}_{4} /$ polymer core/shell/shell nanoparticles with significant enhancement of upconversion fluorescence. Chem Mater 19: pp341-343, 2007.

21. Shan J and Ju Y: A single-step synthesis and the kinetic mechanism for monodisperse and hexagonal-phase NaYF4:Yb, Er upconversion nanophosphors. Nanotechnology 20: 275603, 2009.

22. Wang $\mathrm{L}$ and $\mathrm{Li} \mathrm{Y}: \mathrm{Na}(\mathrm{Y} 1.5 \mathrm{Na} 0.5) \mathrm{F} 6$ single-crystal nanorods as multicolor luminescent materials. Nano Lett 6: 1645-1649, 2006.

23. Wang J, Wang F, Xu J, Wang Y, Liu Y, Chen X, Chen H and Liu X: Lanthanide-doped $\mathrm{LiYF}_{4}$ nanoparticles: Synthesis and multicolor upconversion tuning. Comptes Rendus Chimie 13: 731-736, 2010.

24. Tapmeier TT, Moshnikova A, Beech J, Allen D, Kinchesh P, Smart S, Harris A, McIntyre A, Engelman DM, Andreev OA, et al: The $\mathrm{pH}$ low insertion peptide pHLIP variant 3 as a novel marker of acidic malignant lesions. Proc Natl Acad Sci USA 112: 9710-9715, 2015.

25. Kimbrough CW, Khanal A, Zeiderman M, Khanal BR, Burton NC, McMasters KM, Vickers SM, Grizzle WE and McNally LR: Targeting acidity in pancreatic adenocarcinoma: Multispectral optoacoustic tomography detects $\mathrm{pH}$-low insertion peptide probes in vivo. Clin Cancer Res 21: 4576-4585, 2015.

26. Wagner E: Tumor-targeted delivery of anti-microRNA for cancer therapy: pHLIP is Key. Angew Chem Int Ed Engl 54: 5824-5826, 2015.

27. Liotta LA and Kohn EC: The microenvironment of the tumour-host interface. Nature 411: 375-379, 2001.

28. Raghunand N, Gatenby RA and Gillies RJ: Microenvironmental and cellular consequences of altered blood flow in tumours. Br J Radiol 76 Spec No 1: S11-S22, 2003.

This work is licensed under a Creative Commons Attribution-NonCommercial-NoDerivatives 4.0 International (CC BY-NC-ND 4.0) License. 